\title{
10. Der gegenwärtige Stand und was zu tun bleibt
}

Durch den Erfolg in Schönbühel ermutigt beschlossen die Mitarbeiter der Grazer FDÖP im Jahr 2005, die Bestände an der Houghton Library neuerlich vollständig durchzusehen und sowohl das Verzeichnis der Manuskripte von Mayer-Hillebrand als auch Houghtons Correspondence Guide für den wissenschaftlichen Briefwechsel Brentanos anhand der Originale zu überprüfen. Diese Überprüfung förderte zwar eine große Zahl von Ungenauigkeiten und Fehlern zutage, zu wirklich neuen Entdeckungen, die mit denen in Schönbühel vergleichbar gewesen wären, kam es jedoch nicht. Bei dieser Revision wurden vom Verf. auch die Dokumente in den beiden Kartons, die bis 1990 gesperrt gewesen waren, durchgesehen und erstmals katalogisiert. ${ }^{1}$ Im Anschluss an die Revisionsarbeiten wurde der gesamte Bestand an der Houghton Library in Zusammenarbeit mit den Imaging Services der Harvard College Library und dem Manuscript Department der Houghton Library digitalisiert. ${ }^{2}$ Dieses zur Gänze von der Franz Brentano Foundation Boston finanzierte Projekt konnte aufgrund der schieren Masse des Materials erst im Jahr 2010 abgeschlossen werden. Wie schon im Falle Schönbühels wurden die Bilddateien an der FDÖP verwaltet. Seit 2015 sind sie fast vollständig online zugänglich. ${ }^{3}$

Ebenfalls im Jahr 2005 gelang es der FDÖP den Kontakt mit Ursula Brentano aufzunehmen, die in Blonay das Archiv der Familie Brentano verwaltet. Dank ihres freundlichen Entgegenkommens war es dem Verf. möglich, in den Jahren 2005 und 2008 ein provisorisches Verzeichnis dieser Sammlung herzustellen. Im Familienarchiv werden neben dem Nachlass von Gio und Sofie Brentano rund zwanzigtausend Seiten an Originaldokumenten aufbewahrt, die auf Franz Brentano zurückgehen: Neben mehr als viertausend Briefen ${ }^{4}$ und dem gesamten poetischen Schaffen ${ }^{5}$ findet sich auch philosophisch Bedeutsames, etwa eine von Brentano handschriftlich annotierte Korrekturfahne der PeS.

1 Vgl. dazu oben 313, Fn. 19.

2 Die digitalen Faksimiles wurden im Standard-Archivformat tiff mit 300 dpi Auflösung (z.T. sogar mit 600 dpi) und 24 bit Farbtiefe hergestellt.

3 Vgl. dazu unten 321.

4 Darunter die an biographischer Bedeutsamkeit kaum zu übertreffenden Briefwechsel Brentanos mit seiner Mutter und seiner Schwester Claudine, und ein Teil der Korrespondenz mit seinem Bruder Lujo.

5 Brentanos poetisches Schaffen umfasst mehrere hundert Dichtungen von unterschiedlichem Umfang. Für die Qualität dieser Arbeiten spricht immerhin, dass schon die mit Brentano befreundete Dichterin Marie von Ebner-Eschenbach ihn aufforderte, diese zu veröffentlichen. 
Im Jahr 2009 wurde das Franz Brentano-Archiv Graz (FBAG) gegründet, zunächst als Teil der FDÖP. Nachdem die FDÖP 2011 aus politischen Gründen als eigenständige Forschungsinstitution $\mathrm{zu}$ existieren aufgehört hatte, wurde das FBAG in das philosophische Institut (Fachbereich Geschichte der Philosophie) der Karl Franzens-Universität integriert. Neben den von der Brown University übernommenen Dokumenten und den Nachlässen von Katkov und Chisholm verfügte das FBAG nun auch über die digitalen Faksimiles des Kastil-Nachlasses und über eine vollständige digitale Kopie der Brentano-Sammlung der Houghton Library. Im Jahr 2012 kam es zu einer weiteren Vergrößerung der Brentano-Sammlung. Schon 2011 hatte nämlich Robert Petre, der Archivar des Oriel College der Oxford University, das FBAG darüber informiert, dass er bei Revisionsarbeiten auf Dokumente gestoßen sei, die mit Franz Brentano und seiner Schule in Zusammenhang stünden. Da diese Dokumente nicht den Beständen der College Library zugeordnet werden konnten, bot er sie im Auftrag des College dem FBAG als Schenkung an. ${ }^{6}$ Als diese Dokumente schließlich ein Jahr später in Graz eintrafen, wurde schnell klar, dass es sich um jene „unwichtigen Manuskripte“ handelte, die 1941 vom Hauptbestand des Brentano-Nachlasses getrennt und schließlich bei dessen Übersendung in die USA vergessen worden waren. Das Konvolut der „unwichtigen“ Schriften enthielt mehr als dreißig zum Teil umfangreiche Werkmanuskripte Brentanos sowie den größten Teil der Briefe von Stumpf und Kraus an Brentano. ${ }^{7}$

Ein weiterer Zuwachs zu den Beständen des FBAG fällt in die allerjüngste Zeit. Im Herbst 2015 wurde das ehemalige Brentano-Haus in Schönbühel von der Familie Kastil an das Schlossgut Schönbühel-Aggsbach verkauft, dass das Gebäude mittlerweile in ein Wohnhaus umgebaut hat - das Schönbüheler Brentanohaus ist damit endgültig Vergangenheit. Die Gutsverwaltung, in deren Besitz auch der Kastil-Nachlass übergegangen war, stellte diesen 2016 dem FBAG zur Verfügung. Damit haben sich - abgesehen von einigen kleineren Sammlungen ${ }^{8}$ - die Standorte des Brentano-Nachlasses auf drei große Sammlungen reduziert: auf die Houghton Library, auf das FBAG und auf das Archiv der Familie Brentano in

6 Vgl. dazu oben 284.

7 Bei den Manuskripten zu Erkenntnistheorie und Logik (12), zur Metaphysik (2) und zur Naturwissenschaft (5) handelt es sich um solche, die in Mayer-Hillebrands Katalog als „fehlend“ ausgewiesen sind, also immerhin bereits bekannt waren; 15 weitere Manuskripte - darunter ein Fragment aus Brentanos Würzburger Metaphysikvorlesung - scheinen jedoch auf keiner der bekannten Listen auf. Weiters enthielt das Konvolut 121 Briefe von Stumpf, 130 Briefe von Kraus, die Briefe von John Stuart Mill an Brentano sowie zahlreiche Prager Abschriften.

8 Erwähnenswert sind hier vor allem das Freie Deutsche Hochstift in Frankfurt und das Stadtund Stiftsarchiv Aschaffenburg. Zu einer Auflistung der bekannten Standorte vgl. unten den Anhang 8, 468-470. 
Blonay. Obwohl über das Schicksal des Letzteren noch keine endgültige Entscheidung gefallen ist, lässt sich nun erstmals in der hundertjährigen Geschichte des Nachlasses von Brentano ein - wenn auch zum Teil nur provisorischer Überblick geben über die Gesamtheit dieses kulturhistorischen Denkmals ersten Ranges. Dass möglicherweise noch hier und dort ein Brief von oder an Brentano auftauchen mag, ändert daran wenig. Durch die neuen Möglichkeiten der Digitalisierung erhält die Brentano-Forschung nun einen - im Vergleich zu den Mikrofilmen - wesentlich einfacheren Zugang auch zu dem, was Brentano in seiner „langen literarischen Zurückgezogenheit“ produziert hat. Auf der Website des FBAG sind mittlerweile fast alle Werkmanuskripte und wissenschaftlichen Korrespondenzen, deren Originale sich an der Houghton Library befinden, online als digitale Faksimiles, ergänzt durch die Metadaten der einzelnen Dokumente, zugänglich. ${ }^{9}$ Die so geschaffene Infrastruktur erlaubt es, auch die restlichen Sammlungen, sobald diese in Datenbanken erfasst und digitalisiert sind, sukzessive in das Archiv zu integrieren.

Mit der Zugänglichmachung des gesamten Nachlasses ist natürlich nur eine erste Voraussetzung für eine neue und fundiertere Bewertung der Philosophie Brentanos geschaffen. Ein weiterer wichtiger Schritt wäre eine völlige Neukatalogisierung des Gesamtnachlasses, die alleine schon dadurch angebracht erscheint, dass nun auch die mehrfach erweiterte Sammlung des FBAG und - mit gewissen Einschränkungen - das Familienarchiv mit einbezogen werden können. Bei dieser Gelegenheit sollten die Werkmanuskripte, selbst wenn man sie in den bisherigen thematischen Abteilungen belässt, so weit als möglich in eine chronologische Ordnung gebracht werden. Auch gibt es in Mayer-Hillebrands Katalog zahlreiche Konvolute, die in Einzeldokumente aufzulösen und auch einzeln zu erfassen wären. ${ }^{10}$

Die digitalen Faksimiles können - anders als die Mikrofilme - die Originalmanuskripte weitestgehend als primäre Quelle der Forschung ersetzen, aber sie machen natürlich eine Edition keineswegs überflüssig. Somit stellt sich die Editionsproblematik als die größte verbleibende Herausforderung für die Brentano-

9 Der Ausbau des digitalen Archives war Ende 2015 so weit gediehen, dass nur noch die Werkmanuskripte zu Aristoteles und jene Familienbriefe, die bis 1990 gesperrt waren, fehlen. Das digitale Brentano-Archiv wurde von 2013 bis 2015 in einer Kooperation des FBAG mit Hubert Stigler und seinem Zentrum für Informationsmodellierung - Austrian Centre for Digital Humanities geschaffen. Die Software, die dem Archiv die Architektur liefert, ist das von Stigler entwickelte Content Management System GAMS.

10 Beispiele für solche Manuskriptkonvolute sind etwa Ps 67 („Abbrüche aus dem Psychologiekolleg“), Ps 69 („Abbrüche aus den Kollegien über Psychologie aus der Zeit, als Brentano noch an den Irrealia festhielt“), Ps 70 („Zum Psychologiekolleg (Vorarbeiten)“) oder Th 32 („Material zum Band ,Vom Dasein Gottes““). 
Forschung heraus. Zwar waren die zuletzt in Meiners Philosophischer Bibliothek veröffentlichten Bände aus dem Nachlass hinsichtlich der in ihnen angewendeten editorischen Methoden ein beträchtlicher Fortschritt; ${ }^{11}$ für alle Werkmanuskripte Brentanos, die in den von Kraus, Kastil, Mayer-Hillebrand und auch noch von Chisholm herausgegebenen Nachlassbänden versammelt sind, muss jedoch die Forderung nach neuen Editionen erhoben werden, die sich eines textkritischen Verfahrens bedienen und der Entwicklung von Brentanos philosophischem Denken neutral gegenüberstehen, d.h. alle Schriften, unabhängig vom Zeitpunkt ihres Entstehens, mit derselben editorischen Sorgfalt behandeln. Seit Werles Überlegungen zu einer Neuedition von Brentanos Schriften ist man hier bedauerlicherweise keinen wesentlichen Schritt vorangekommen. So warten vor allem die großen Vorlesungen über Psychologie, Metaphysik, Logik und Ethik noch immer auf eine adäquate Edition. Die einzige Ausnahme bildet die schon erwähnte (provisorische) Edition der Logikvorlesung EL 80, die Rollinger vorgelegt hat und die zweifellos einen ersten Schritt in die richtige Richtung bedeutet. ${ }^{12} \mathrm{Er}$ hat auch auf prägnante Weise einige der Probleme beschrieben, die auf die zukünftigen Herausgeber von Brentanos Vorlesungen warten:

Da Brentano die Logik über einen erheblichen Zeitraum für seine Vorlesungen benutzt hat, weist das Manuskript verschiedene Bearbeitungsschritte auf. Es gibt ebenso Blätter in anderen Manuskriptkonvoluten (z.B. EL 81), die zumindest teilweise dem Material in EL 80 angehört haben. Diese unterschiedlichen Schichten der Bearbeitung stellen den Herausgeber vor die größten Probleme. [...] Das Problem einer Edition der Logik wird noch dadurch erschwert, dass Brentano den Text oft nicht durch Einfügung neuer Seiten (die möglicherweise alte ersetzen) revidiert, sondern indem er ausführlich auf die alten Blätter schreibt, was immer wieder dazu führt, dass die neue Textschicht die alte direkt überlagert. Es gibt in der Tat einige Manuskriptseiten für die Logik, die auf diese Weise so dicht bearbeitet sind, dass sie nicht mehr sinnvoll ediert werden können. ${ }^{13}$

Schon Kraus plante für die Ethikvorlesung eine Edition, die diese unterschiedlichen Textschichten sichtbar machen sollte. ${ }^{14}$ Mit dem Einsatz von leistungsfähigen geisteswissenschaftlichen Auszeichnungssystemen wie TEI-XML ließe sich eine solche „Schichten-Edition“ durchaus realisieren; die dafür benötigten Res-

11 Gemeint sind hier die von Klaus Hedwig (Brentano 1980b, Brentano 1987) und Rolf George (Brentano 1986) edierten Bände.

12 Diese Edition, die auf der Basis von TEI-XML erstellt wurde, verlinkt die digitalen Faksimile mit einer diplomatischen (also möglichst genauen textkritischen) und einer normalisierten (Lese-) Fassung der Vorlesung. Sie ist auf der Website des FBAG frei zugänglich (http://gams.unigraz.at/archive/objects/context:bag/methods/sdef:Context/get?mode=edition).

13 ebda.

14 Vgl. oben 270. 
sourcen wären aber vermutlich enorm. Da darüber hinaus für die Brentano-Edition ein den Husserl-Archiven vergleichbarer institutioneller Rahmen fehlt, wird es wohl in absehbarer Zukunft bei Einzelprojekten bleiben, bei denen natürlich auch die Befolgung einheitlicher editorischer Standards nur schwer durchzusetzen sein wird.

Ein letztes Desiderat der Brentano-Forschung bleibt natürlich eine intellektuelle Biographie Brentanos. Wir können hier nur wiederholen, was Kastil dazu vor mehr als einem halben Jahrhundert festgestellt hat:

An einer ihres Gegenstandes würdigen Biographie Franz Brentanos fehlt es. Die Aufgabe harrt der nicht eben wahrscheinlichen Verbindung von vollem Verständnis für seine wissenschaftliche Größe mit hohem schriftstellerischen Können. ${ }^{15}$

Auch Kastil selbst, dem ja in Schönbühel die für eine Biographie wertvollsten Quellen zur Verfügung standen, fühlte sich dieser Aufgabe offenbar nicht gewachsen. Was dazu im ersten Teil dieser Arbeit aufgeschrieben wurde, kann bestenfalls als eine weitere Vorarbeit dazu betrachtet werden. Erst wenn das Archiv der Familie Brentano in vollem Umfang zugänglich sein wird, kann dieses Projekt ernsthaft ins Auge gefasst werden.

15 Kastil 1951, 9. 
